\title{
An Examination of the Factors Contributing to Poor Communication Outside the Physician-Patient Sphere
}

\author{
Shaurya Taran*
}

\begin{abstract}
Health care workers today acknowledge that poor communication is perhaps one of the most prevalent problems in medicine. A number of studies have looked into the causes and outcomes of poor communication in medical facilities, and it appears from these studies that the problem is pressing enough to warrant the attention of not just health care workers, but also the general public. One study conducted in the late 1990s found that poor communication was responsible for causing between 44000 and 98000 patient deaths annually in American hospitals alone. Other studies found that poor communication was one of the leading causes of preventable deaths in hospitals. These worrying reports have spurred a number of worldwide efforts to exhaustively characterize the factors that lead to poor communication between physicians and their patients; by contrast, there has been comparatively less emphasis on analyzing poor communication outside the sphere of physician-patient interactions. In other words, poor communication between physicians and other physicians; between physicians and nurses; and between hospitals and other hospitals, has remained largely unexplored. The relative paucity of papers in these areas is due in part to the difficulty of conducting the necessary large-scale assessments that these topics of research require. As a result, the public remains largely unaware of the few findings that have been published in the literature. It is therefore crucial that the results of these studies be brought to a larger audience so that the issue
\end{abstract}

\footnotetext{
*To whom correspondence should be addressed: Shaurya Taran

McGill University

Telephone: (514) 260-8546

Email: shaurya.taran@mail.mcgill.ca
}

of poor communication in hospitals can be properly appreciated in all of its dimensions.

Independent authors and health care professionals including Daniel Goleman (author of the influential Emotional Intelligence) and Barbara Korsch, respectively, made some of the first pointed attempts to bring the issue of poor communication in hospitals to the public's awareness. Their work prompted general discussions in the medical community on how best to combat the problem of poor communication. Research groups began to rigorously evaluate the claim that healthy physicianpatient dialogue could promote strong rapport between the two parties, while its counterpart, namely poor communication, could lead to hostility and mutual distrust between them. Today, largely because of the independent work of Goleman and Korsch, health care professionals recognize the importance of effective communication in hospitals. However, this recognition has only spurred policy changes in certain areas of healthcare; in other areas, poor communication remains as prevalent now as it did before the seminal work of Goleman and Korsch appeared in print.

One of the areas where communication can at times be deficient is in physician-physician interactions. Poor communication, when it manifests between physicians responsible for the care of the same patient, can significantly hamper medical progress. Perhaps this is not surprising, given that poor communication retards advancement in any collaborative effort; however, researchers were surprised to find just by how much poor physicianphysician communication retarded progress in the treatment of patients. One important study in this area looked at communication between primary 
care physicians and subspecialty consultants. Analysts found that while almost all primary care physicians provided clear background information of their patients to consultants, a large percentage of primary care physicians failed to specify the exact reason for referral to consultants. Furthermore, only a very small percentage of primary care physicians engaged in direct contact with subspecialty consultants. Finally, a staggering percentage of consultants completely neglected to notify primary care physicians of the results of their findings (1). An important implication of these results is that the likelihood for poor communication to develop increases with the number of physicians that are involved in caring for a patient at non-overlapping stages of treatment. As it can be imagined, every poorly written, incomplete, or deficient referral poses the risk of introducing a complication into the patient's condition. In some instances these complications manifest themselves early and can therefore be easily detected and corrected; however, complications can also go unnoticed until it is simply too late to treat them. Complications of the latter type are, contrary to popular belief, quite common in medicine-which is why addressing deficient communication between physicians should be one of the primary focuses of health care policy reformists.

Another primary focus of health research groups should be on characterizing the factors involved in poor communication between residents and attending physicians. Although several papers have given compelling reasons to suggest that this issue is still quite prevalent in teaching hospitals, relatively few studies have rigorously examined the problem. One major study has, however, published its findings on the topic. This study has shown that poor communication between residents and attending physicians can often develop if residents feel that they are being pressed to perform perfectly by their supervisors. (This raises several important questions: to what extent should hospitals encourage their residents to see medical errors as tantamount to crimes? Is such a drastic policy necessary to enforce the idea that errors should be avoided at all costs? Can the same point also be made in a way that does not encourage a fear-based perfectionism? These questions, interestingly, were the focus of another paper. This paper argued that the need to function without error in fact made it much more difficult for physicians to offer highquality care to their patients (2)). Given the unique nature of the resident-attending physician relationship, perhaps it is not surprising to see why such an area might be a likely site for communication breakdown. Although residents are instructed to ask attending physicians for advice and assistance in difficult or medically ambiguous situations, the reality is that residents oftentimes refrain from asking the appropriate questions or requesting the appropriate advice for fear of being perceived as medically incompetent. Residents may further abstain from seeking the guidance of attending physicians if they feel that they should "know" how to do something on their own. In other instances, residents simply don't wish to be a nuisance to their attending physicians (one frequently given example of this involves residents on night shifts declining to wake up their supervisors-even if they are uncertain of which course of action to take with a particular patient). Communication breakdown, and sometimes even a complete communication failure, can also result between residents and attending physicians if residents feel uncomfortable questioning the decisions of their supervisors. Oftentimes residents' hesitancy in these situations is due in part to their desire not to offend attending physicians. This fear leads to the suppression of potentially legitimate doubts and concerns on how best to handle a particular situation; as a result of the communication breakdown, patients may suffer (3).

Poor communication, as suggested by the above paper, tends to evolve out of the inevitable and irreversible hierarchy of power within hospitals. Indeed, the very existence of hierarchies in hospitals tends to increase the likelihood of poor communication developing at some level or another within the vertical power structure. This is not to suggest that the hierarchical organization of power in hospitals is a bad thing -it is only meant to imply that there are strong potentials for poor communication to develop in multiple places within the medical hierarchy. One of these places is between physicians and nurses. Indeed, just as residents feel occasionally unwilling to ask for the help of attending physicians, so too do nurses frequently refrain from asking physicians potentially "obvious" or unimportant questions (3). Given that both physicians and nurses are intimately involved in the care and support of patients, major communication breakdown between these two parties could potentially translate into serious medical difficulties for their patients. One important study found that this problem was exacerbated in certain instances, such as when physicians instructed nurses to convey important medical information to patients rather than taking on that responsibility themselves (4). 
Another study showed that poor communication between physicians and nurses often developed when physicians relied on written orders to convey instructions to nurses (3). The study found that physicians routinely wrote important details into patients' medical files-often these details included crucial instructions for nurses, such as when to administer particular treatments to patients. If nurses missed checking patients' medical files, they consequently also missed the important instructions that were written in them. This raises the crucial question of why physicians continue to employ indirect modes of communication to the extent they do, and why nurses don't object to it (once again, this is not to suggest that written communication be entirely prohibited, but that written communication is, in most respects, a poor substitute for direct verbal communication). Perhaps one reason why written communication remains prevalent is that nurses fear questioning what they might consider to be a standard practice among all physicians. Here again it can be seen that the reluctance to question the practices of superiors may lead to a communication breakdown-or, more seriously, to a complete communication failure.

Just as serious as the complete failure of communication is the problem of delayed communication. Not surprisingly, delayed communication between physicians significantly hampers medical progress; for this reason, it is often classified under the broader category of poor communication. The proper orchestration of multiple events by multiple physicians depends, perhaps first and foremost, on the timely transmission of information between physicians. Instances in which this communication is delayed can often result in detrimental consequences. For example, one study found that patients were sometimes released from hospitals before all of their medical tests had even been completed (5). This particular study attributed the premature discharge of patients to delayed or ineffective communication between inpatient and outpatient physicians. Although in most cases results from pending tests were negative, in several instances pending test results were potentially actionable. For this reason, it is perhaps easy to see why timely, effective communication between inpatient and outpatient physicians is necessary at all steps in the medical process. A breakdown or delay in communication could-and, in fact, quite frequently does-lead to the development of complications in the health of patients following their premature discharge.
The challenges of finding solutions to the poor communication problem when it manifests outside the sphere of physician-patient interactions are numerous; nevertheless, several important papers have proposed some simple, practical, and occasionally innovative solutions to the problem.

Concerning poor communication between referring physicians and subspecialty consultants, one paper suggested that both parties limit indirect communication and instead engage in direct verbal communication whenever possible (1). This can be difficult (if not entirely impossible) if the two physicians belong to different clinics or hospitals. In such cases communication over telephones could be helpful; however, this is not a standard practice among physicians, and past efforts to promote telephone communication have met with little success. One recent paper proposed an interesting alternative to communicating over telephones: teleconferencing. Researchers looked into the effectiveness of teleconferencing as a means of communicating patient information; they found that physicians generally responded well to the new technology, and had little difficulty in acquainting themselves with it (6). The teleconferencing equipment itself was easy to set up and operated with a minimum of technical difficulties, which added to its appeal. Video capabilities of the teleconferencing software allowed physicians to communicate visual information, such as the results of a dermatological test, with relative ease (the quality of these images, however, was in some instances inadequate). In preliminary trials, satisfaction among first-time users of the new technology was fairly high. This study did, however, acknowledge the limitations of teleconferencing, and pointed out that such an alternative was unlikely to find its way into mainstream medicine in the near future. Other studies have provided simpler methods to increase direct communication between two physicians. One of the proposed suggestions involves creating smaller administrative units within larger hospitals to bring primary and secondary physicians into closer proximity to each other (7). If direct contact is simply not possible, such as when two physicians belong to different hospitals or clinics, then simple things such as aesthetic modifications to the referral letter format could, one study suggests, promote better communication between the two parties (7). The relatively simple nature of the above solutions suggests that it should not be difficult to increase direct communication between physicians, provided that some simple and practical modifications are made in the way that medical 
information is communicated among health care professionals.

There are, however, numerous difficulties in addressing poor communication in other areas. For example, there is simply no systemized way to promote healthier communication between residents and attending physicians, or between physicians and nurses. It is perhaps easy to see why this is: Communication failures in these areas arise largely when one party fears questioning the authority or medical ability of a superior party. Hospitals have always encouraged residents and nurses to raise their concerns whenever appropriate, but the fact remains that individuals on the lower levels of the hierarchy will inevitably feel intimidated by individuals on the upper levels-which, as a consequence, causes residents and nurses to suppress instead of voice their concerns. Indeed, there is no simple solution to the poor communication problem when it manifests in the interactions of residents and attending physicians, and nurses and physicians. This could perhaps be one area of study of future papers.

Poor communication, as suggested by the collection of above studies, appears readily in interactions outside the physician-patient sphere. Given the number of places where this problem can manifest-physician-physician interactions, resident-attending physician interactions, and physician-nurse interactions, to give a few examples-it is somewhat surprising to see that poor communication in these areas received disproportionately little attention until about the mid- 1980s. Today, however, health care analysts generally acknowledge the need to completely characterize the factors contributing to poor communication when it manifests between medical workers themselves. Since this is still a relatively new undertaking, the general public remains largely unaware of the problem. Nevertheless, small initiatives can still be taken to bring the larger issue of poor communication in hospitalswith all of its grim implications and uncomfortable ramifications-into the public discourse.

\section{REFERENCES}

1. McPhee SJ, Lo B, Saika GY, et al. How Good Is Communication Between Primary Care Physicians and Subspecialty Consultants? Arch Intern Med. 1984; 144(6):1265-1268.

2. Leval MR. Human Factors and Surgical Outcomes: A Cartesian Dream. The Lancet. 1997; 349 (9053): 723-725

3. Sutcliffe KM, Lewton E, Rosenthal MM. Communication Failures: An Insidious Contributor to Medical Mishaps. Acad Med. 2004; 79(2): 186-194.

4. Fallowfield L, Jenkins V. Effective Communication Skills are the Key to Good Cancer Care. European Journal of Cancer 1999; 35(11):1592-1597.

5. Roy CL, Poon EG, Karson AS, et al. Improving Patient Care: Patient Safety Concerns Arising from Test Results That Return after Hospital Discharge. Ann Intern Med 2005 143 (2):121-128

6. Harrison R, Clayton W, Wallace P. Can Telemedicine be Used to Improve Communication Between Primary and Secondary Care? Brit Med Jour. 1996; 313 (7069):13771380

7. Epstein RM. Communication Between Primary Care Physicians and Consultants Arch Fam Med. 1995; 4(5):403-409. 